\title{
Risk factors for mortality and progression to severe COVID-19 disease in the Southeast region in the United States: A report from the SEUS Study Group ${ }^{a}$
}

\author{
Athena L. V. Hobbs PharmD ${ }^{1}$ (1) Nicholas Turner MD², Imad Omer MD³, Morgan K. Walker MD4, Ronald M. Beaulieu MD ${ }^{5}$, \\ Muhammad Sheikh $M D^{3}$, S. Shaefer Spires $M D^{2}$, Christina T. Fiske $M D^{5}$, Ryan Dare $M D^{6}$, Salil Goorha $\mathrm{MD}^{3}$, \\ Priyenka Thapa $\mathrm{MD}^{6}$, John Gnann $\mathrm{MD}^{7}$, Jeffrey Wright $\mathrm{MD}^{3}$ and George E. Nelson $\mathrm{MD}^{5}$ \\ ${ }^{1}$ Department of Pharmacy, Methodist University Hospital, Memphis, Tennessee, ${ }^{2}$ Department of Medicine, Duke University Medical Center, Durham, North \\ Carolina, ${ }^{3}$ Department of Medicine, Baptist Memorial Hospital-Memphis, Tennessee, ${ }^{4}$ Department of Medicine, Louisiana State University Health Sciences Center, \\ New Orleans, Louisiana, ${ }^{5}$ Department of Medicine, Vanderbilt University Medical Center, Nashville, Tennessee, ${ }^{6}$ Department of Medicine, University of Arkansas \\ for Medical Sciences Medical Center, Little Rock, Arkansas and ${ }^{7}$ Department of Medicine, The Medical University of South Carolina University Medical Center, \\ Charleston, South Carolina
}

\begin{abstract}
Objective: Identify risk factors that could increase progression to severe disease and mortality in hospitalized SARS-CoV-2 patients in the Southeast region of the United States.

Design, setting, and participants: Multicenter, retrospective cohort including 502 adults hospitalized with laboratory-confirmed COVID-19 between March 1, 2020, and May 8, 2020 within 1 of 15 participating hospitals in 5 health systems across 5 states in the Southeast United States.

Methods: The study objectives were to identify risk factors that could increase progression to hospital mortality and severe disease (defined as a composite of intensive care unit admission or requirement of mechanical ventilation) in hospitalized SARS-CoV-2 patients in the Southeast United States.

Results: In total, 502 patients were included, and 476 of 502 (95\%) had clinically evaluable outcomes. The hospital mortality rate was $16 \%$ (76 of 476); 35\% (177 of 502) required ICU admission and 18\% (91 of 502) required mechanical ventilation. By both univariate and adjusted multivariate analyses, hospital mortality was independently associated with age (adjusted odds ratio [aOR], 2.03 for each decade increase; $95 \%$ confidence interval [CI], 1.56--2.69), male sex (aOR, 2.44; 95\% CI, 1.34-4.59), and cardiovascular disease (aOR, 2.16; 95\% CI, 1.15-4.09). As with mortality, risk of severe disease was independently associated with age (aOR, 1.17 for each decade increase; $95 \% \mathrm{CI}, 1.00-1.37$ ), male sex (aOR, 2.34; 95\% CI, 1.54-3.60), and cardiovascular disease (aOR, 1.77; 95\% CI, 1.09-2.85).
\end{abstract}

Conclusions: In an adjusted multivariate analysis, advanced age, male sex, and cardiovascular disease increased risk of severe disease and mortality in patients with COVID-19 in the Southeast United States. In-hospital mortality risk doubled with each subsequent decade of life.

(Received 18 August 2020; accepted 30 December 2020; electronically published 11 January 2021)

\footnotetext{
Author for correspondence: Athena L. V. Hobbs, E-mail: athena.hobbs@mlh.org aSoutheast United States (SEUS) COVID-19 Outbreak Investigation Team members: The Southeast United States (SEUS) COVID-19 Outbreak Investigation Team members include the following in alphabetical order: Baptist Memorial Healthcare Corporation: Muhammad Akbik, Krista Bachert, Madeline Belk, Allison Brunson, Allie Crawford, Wade Dickerson, Laura Fuller, Benjamin Hampton, Keri Mills, Alyssa Morgan, Musa Mubarez, Nicholson Perkins, Jacob Rigdon, Stephen Threlkeld, Michael Threlkeld, Lindsay White, Kevin VanMaldeghem, Maria Zhorne; Duke University Medical Center: David Stevens, Jenny Wu; Louisiana State University Health Sciences Center: Diana Hamer, Anthony Naquin, Catherine O’Neal, Jason Patel, Blake Savoie.

Cite this article: Hobbs ALV, et al. (2021). Risk factors for mortality and progression to severe COVID-19 disease in the Southeast region in the United States: A report from the SEUS Study Group. Infection Control \& Hospital Epidemiology, 42: 1464-1472, https:// doi.org/10.1017/ice.2020.1435
}

Severe acute respiratory syndrome coronavirus 2 (SARS-CoV-2) and the disease it causes, novel coronavirus disease 2019 (COVID-19), was first identified in Wuhan, China, in December 2019, but it quickly spread and was declared a global pandemic by the World Health Organization (WHO) on March 11, 2020. ${ }^{1,2}$ Several early studies demonstrated various pre-existing conditions to be associated with severe disease ${ }^{3,4}$ and mortality, ${ }^{5,6}$ and in the United States, data have shown that between $14 \%$ and $30 \%$ of hospitalized patients require ICU level care and that older age and male sex associated with increased risk for ICU admission and mortality.,

The Southeast region of the United States reports the highest rates of obesity, diabetes, hypertension, and smoking in the

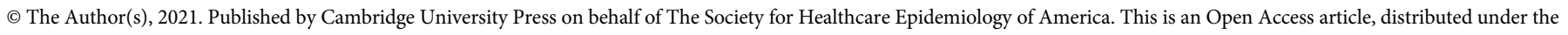

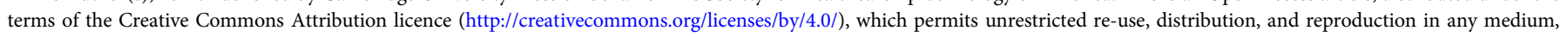
provided the original work is properly cited. 
nation, ${ }^{9-12}$ yet data about the effects of COVID-19 in this region are limited to 2 studies. Both relied on administrative data from a single health system; one study did not evaluate mortality as an outcome and the other reported outcomes that were not unadjusted for underlying comorbidities. ${ }^{13,14}$ A wide, continually expanding range of clinical features have been reported, and continued description of emerging clinical characteristics and outcomes in diverse, underrepresented populations are needed. ${ }^{7,8,15}$

The purpose of this multicenter, retrospective cohort is to determine risk factors associated with severe clinical outcomes from COVID-19 in a patient population with a high prevalence of comorbidities; determining these factors can help clinicians identify and triage high-risk patients at risk for adverse outcomes associated with COVID-19 in the Southeast United States.

\section{Methods}

This multicenter, retrospective cohort study was conducted through the Southeast United States (SEUS) study group, which encompasses 15 hospitals, including both academic and community healthcare settings, ranging from 25 to 1,046 licensed beds within 5 healthcare systems across 5 states: Arkansas, Louisiana, Mississippi, North Carolina, and Tennessee. Adult hospitalized patients with a positive SARS-CoV-2 real-time reverse transcriptasepolymerase chain reaction (RT-PCR) assay using nasopharyngeal swab specimens collected during or immediately prior to hospital admission were identified through a review of positive laboratory values and were enrolled consecutively between March 1, 2020, through May 8, 2020. Demographic data, exposure risk, underlying conditions, clinical presentation, medications, nonmedication therapy (eg, mechanical ventilation), and outcome data were collected for eligible patients through electronic medical record (EMR) review and were entered into a REDCap database. ${ }^{16}$

The primary outcome was in-hospital mortality in patients with COVID-19 in the Southeast United States. Secondary outcomes included severe disease (defined as a composite of ICU admission or requirement of mechanical ventilation) and other clinical characteristics and epidemiological features associated with COVID-19 in the Southeast United States.

\section{Underlying conditions}

Chronic kidney disease was defined as Kidney Disease Outcomes Quality Initiative (KDOQI) stages 3 through 5 for estimated glomerular filtration rate $(\mathrm{eGFR})<60 \mathrm{~mL} / \mathrm{min}$. Peripheral vascular disease included venous stenosis, deep vein thrombosis, and chronic venous stasis. Connective tissue disease included osteoarthritis and rheumatoid arthritis. Liver disease was defined as chronic hepatitis, portal hypertension, or cirrhosis. Although they were evaluated separately, we also analyzed a composite of patients with immune compromising conditions, cardiovascular disease, and pulmonary disease to reduce the impact of a small sample size. Immune compromise included active cancer or hematologic malignancy, history of solid-organ transplantation, HIV/AIDS, or autoimmune diseases with active use of immunosuppressive medications. Cardiovascular disease included congestive heart failure, coronary artery disease, arrhythmia, or other cardiovascular disease. Pulmonary disease was defined as asthma, COPD, or other chronic lung disease.

\section{Statistical analysis}

Descriptive summary data were reported as counts and percentages for categorical variables, and mean ( \pm standard deviation
[SD] for normally distributed) or median (including interquartile range [IQR] for nonnormally distributed) for continuous variables. Outcomes analyses were limited to patients who were either discharged or deceased by the end of the inclusion period (May 8 , 2020). Patients were analyzed across age groups defined in 10-year increments (eg, 20-29 years) through 99 years, accounting for all patients. Comparisons of categorical data between groups were analyzed using the $\chi^{2}$ test or the Fisher exact test when appropriate, and continuous data were analyzed using the Student $t$ test or the Mann-Whitney U test when appropriate. Analysis of variance (ANOVA) was used for comparisons of continuous data across multiple groups when applicable.

We developed 3 separate multivariable logistic regression models to evaluate risk factors for hospital mortality or severe disease and to analyze any association between hospital mortality and treatment with azithromycin or hydroxychloroquine. Full models included all of the following a priori selected clinical variables: age, race, ethnicity, sex, body mass index (BMI), cardiovascular disease, pulmonary disease, liver disease, kidney disease, history of stroke, hypertension, diabetes, active smoking, peripheral vascular disease, connective tissue disease, or immune compromise. For each model, covariates were assessed for collinearity by assuring variance inflation factors $<2$, and continuous variables were assessed for nonlinearity by comparing to a restricted cubic spline model. Potential institution-specific differences in mortality were assessed, and no significant independent difference was detected when included as a covariate. For the treatment effect model, subjects participating in any clinical studies were excluded both to protect study integrity and because receipt of active study drug versus placebo could not be verified. All statistical analyses were conducted with $\mathrm{R}$ version 3.6.0 software (R Foundation for Statistical Computing, Vienna, Austria). All statistical tests were 2-sided with an $\alpha$ level of 0.05 . All confidence intervals (CIs) were $95 \%$.

\section{Results}

In total, 502 adult hospitalized patients were included in the analysis. All COVID-19 cases were index cases and were confirmed by RT-PCR on specimens collected from the nasopharynx. ${ }^{17}$ Median age was 62 years (IQR, 49-71). Of these 502 patients, 225 (45\%) were female; 287 (57\%) were African American (black), 190 (38\%) Caucasian (white), and 21 (4.2\%) reported Hispanic ethnicity (Table 1). Patients were treated in facilities across 5 states in the Southeast: $163(32 \%)$ in Tennessee, 127 (25\%) in Mississippi, $80(16 \%)$ in Louisiana, 76 (15\%) in North Carolina, and $56(11 \%)$ in Arkansas (Table 2). Also, $50 \%$ of patients received hydroxychloroquine at some point during their hospitalization, primarily reflecting the practice of a single health system at the time (Table 1).

Baseline risk factors for COVID-19 included known exposure to a COVID-19-positive individual (199 of 502, 40\%) and presentation from a congregate setting (eg, nursing facility or prison (155 of $502,31 \%)$. Of 502 a COVID-19-positive individuals, only $35(7 \%)$ were healthcare workers; 11 of these $(31 \%)$ were nurses; $6(17 \%)$ were physicians; and 18 (51\%) identified themselves as healthcare workers without designated role. Of these 502 patients 218 (43\%) were evaluated prior to admission, and 133 (61\%) of these 218 were assessed in an emergency department (ED), though 366 of the total 502 patients (73\%) had a positive SARS-CoV-2 RTPCR during admission. The mean time between a positive assay and admission was 0.7 days $( \pm 2.3)$ for those with a positive test prior to admission. Also, 471 (94\%) were symptomatic upon 
Table 1. Baseline Characteristics of Hospitalized Patients with COVID-19 by Disease Severity and Mortality, Southeast United States

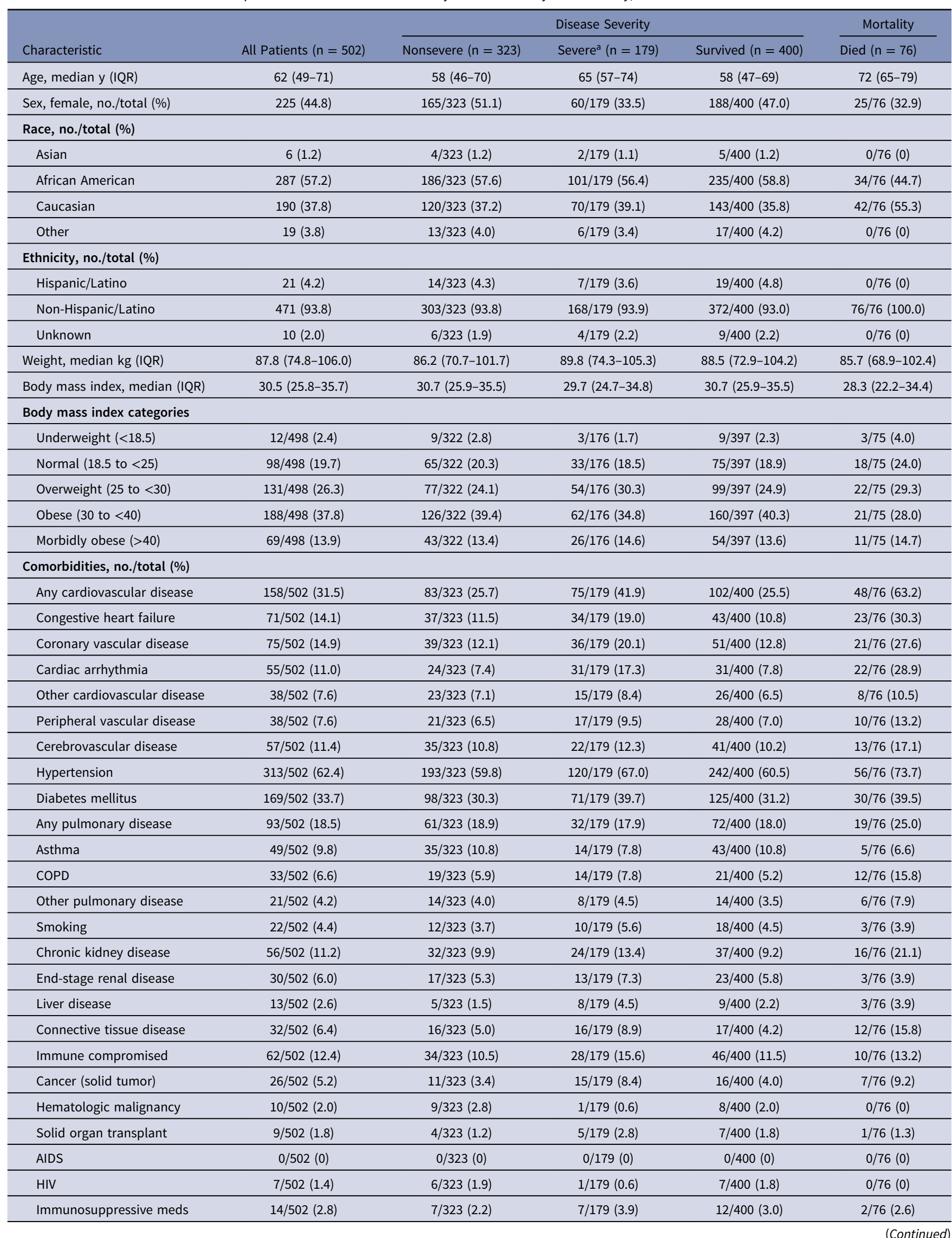


Table 1. (Continued)

\begin{tabular}{|c|c|c|c|c|c|}
\hline \multirow[b]{2}{*}{ Characteristic } & \multirow[b]{2}{*}{ All Patients $(n=502)$} & \multicolumn{3}{|c|}{ Disease Severity } & \multirow{2}{*}{$\frac{\text { Mortality }}{\text { Died }(n=76)}$} \\
\hline & & Nonsevere $(n=323)$ & Severe $^{a}(n=179)$ & Survived $(n=400)$ & \\
\hline \multicolumn{6}{|l|}{ Treatment, no./total. (\%) } \\
\hline Azithromycin & $246 / 496(49.6)$ & $157 / 319(49.2)$ & $89 / 177(50.3)$ & 203/394 (51.5) & $33 / 76(43.4)$ \\
\hline Convalescent plasma & $6 / 496(1.2)$ & $0 / 319(0)$ & $6 / 177(3.4)$ & $1 / 394(0.3)$ & $2 / 76(2.6)$ \\
\hline Hydroxychloroquine & $245 / 490(50.0)$ & $154 / 317(48.6)$ & $91 / 173(52.6)$ & $200 / 390(51.3)$ & $36 / 75(48.0)$ \\
\hline Lopinavir & $0 / 496(0)$ & $0 / 320(0)$ & $0 / 176(0)$ & $0 / 394(0)$ & $0 / 76(0)$ \\
\hline Remdesivir & $6 / 492(1.2)$ & $0 / 316(0)$ & $6 / 176(3.4)$ & $3 / 392(0.8)$ & $0 / 76(0)$ \\
\hline Tocilizumab & $23 / 497(4.6)$ & $3 / 320(0.9)$ & $20 / 177(11.3)$ & $12 / 395(3.0)$ & $8 / 76(10.5)$ \\
\hline Study participant & $23 / 446(5.2)$ & $11 / 285$ (3.9) & $12 / 161(7.5)$ & $16 / 354(4.5)$ & $5 / 66(7.6)$ \\
\hline Length of stay, median d (IQR) & $5.9(3.0-10.1)$ & $4.7(2.5-6.9)$ & $10.0(4.8-15.2)$ & $5.9(2.6-9.2)$ & $7.3(2.8-11.8)$ \\
\hline
\end{tabular}

Note. IQR, interquartile range; COPD, chronic obstructive pulmonary disease; HIV, human immunodeficiency virus; AIDS, acquired immunodeficiency syndrome.

asevere disease, defined as a composite of ICU admission or requirement of mechanical ventilation.

admission. Fever was the most commonly reported symptom $(352$, $70 \%)$, followed by dyspnea $(317,63 \%)$, dry cough $(277,55 \%)$, fatigue $(187,37 \%)$, myalgia $(142,28 \%)$, nausea $(105,21 \%)$, and diarrhea defined as $>3$ episodes of loose stool within 24 hours $(104,21 \%)$. The mean duration of symptom onset prior to admission was $5.2 \pm 4.5$ days (Table 2).

Almost all patients (476 of 502, 95\%) were either discharged or deceased by the end of the inclusion period and were included in outcome analyses. The overall hospital mortality rate was $16 \%$ (76 of 476). Moreover, 177 (35\%) required intensive care unit (ICU) admission, and 91 (18\%) required mechanical ventilation. Hospital length of stay was significantly longer for patients with severe disease (median, 10.0 days; IQR, 4.8-15.2) relative to nonsevere disease (median, 4.7 days; IQR, 2.5-6.9; $P<.01$ ).

Most patients ( 422 of 502, 84\%) had at least 1 comorbidity, and 190 (38\%) had at least 3 comorbidities. Hypertension was the most common comorbidity, affecting 313 patients (62\%) More than half ( 257 of $498,52 \%$ ) were obese, with a median admission weight of $87.8 \mathrm{~kg}$ (IQR, 74.8-106.0) and median BMI of 30.5 (IQR, 25.835.7). One-third of patients had been diagnosed with diabetes mellitus (169 of 502, 34\%), followed by coronary vascular disease and congestive heart failure, which affected 75 patients $(15 \%)$ and 71 patients (14\%), respectively. Notably, only 49 (10\%) of patients had asthma, $33(7 \%)$ had COPD, 22 (4\%) were active smokers, and $21(4 \%)$ had another chronic pulmonary disease (Table 1).

Hospital mortality was independently associated with age (adjusted odds ratio [aOR], 2.03 for each decade increase in age; 95\% confidence interval [CI], 1.56-2.69), male sex (aOR, 2.44; 95\% CI, 1.34-4.59), cardiovascular disease (aOR, 2.16; 95\% CI, 1.15-4.09), and connective tissue disease (aOR, 3.13; 95\% CI, $1.25-7.81$ ) by both univariate and adjusted analysis. Each comorbidity was individually assessed in univariate analysis; however, any statistical significance was lost after adjusting for additional comorbidities (Table 3 and Supplementary Tables S1 and S2 online). Patients at the extremes of both age and BMI tended to have higher mortality rates. For example, individuals aged 21-40 years and 61-80 years had higher mortality rates if they also had a BMI > 40 ( 1 of $13,7.7 \%$ and 9 of $23,39.1 \%$ respectively; Table 4). Similarly, mortality rates among individuals over the age of 80 appeared to be higher for underweight individuals versus those with a BMI between 18.5 and $<25$ ( 3 of $5,60.0 \%$ vs 8 of 21 , $38.1 \%)$. Small numbers of individuals were included in the most extreme combinations of age and BMI, which could have prevented comparisons from reaching statistical significance (age $\times \mathrm{BMI}$ interaction test, $P=.10$ ) (Table 4). Neither azithromycin (aOR, 0.69; 95\% CI, 0.35-1.37) nor hydroxychloroquine (aOR, 1.52 ; $95 \%$ CI, 0.77-3.06) were associated with any significant reduction in mortality by univariate or adjusted analysis (Table 1). Analysis was limited to these therapeutic agents due to limited availability of alternatives during the study period. Risk of severe disease, as with mortality, was independently associated with age (aOR, 1.17 for each decade increase in age; 95\% CI, 1.00-1.37), male sex (aOR, 2.34; 95\% CI, 1.54-3.60), and cardiovascular disease (aOR, 1.77; 95\% CI 1.09-2.85) (Table 5). We also included a Kaplan-Meier mortality curve stratified by disease severity as a time to event analysis (Fig. 1).

\section{Discussion}

This multicenter study is the first to report risk factors for severe disease and death in hospitalized patients with COVID19 in the Southeast region of the United States, which encompasses a patient population with high rates of underlying comorbidities. Patients included in these analyses represent a large geographic region underreported in current studies and hospitalized in both community and academic institutions from 5 separate health systems in 5 different states in the Southeast. This broad representation, in addition to extensive chart review, allowed for in-depth, patientlevel detail with adjusted risk factor analyses not currently available in many larger-scale epidemiological studies using administrative data sets that often have high rates of missing data.

In a multivariate analysis, age emerged as an independent risk factor for in-hospital mortality and severe COVID-19 disease with mortality risk doubling with each consecutive decade of life. Age was also an independent risk factor for severe disease, increasing 1.17 fold (95\% CI, 1.00-1.37; $P=.05$ ) for each subsequent decade. These findings are consistent with recent observational cohort studies. ${ }^{6,18-20}$ However, despite almost half of our cohort being $\leq 60$ years, only 8 deaths occurred in this group, resulting in a lower mortality rate in this younger cohort compared to earlier reports. ${ }^{7,20}$ Furthermore, few patients $(n=68)$ were $\leq 40$ years, which accounted for only 1 death. We identified age as an important prognostic factor for worse outcomes and compounded risk with increasing age in patients with COVID-19 using a robust risk assessment based on 3 different multivariate statistical models that accounted for a population with multiple comorbidities and diverse demographics. 
Table 2. Presentation Characteristics of Hospitalized Patients with COVID-19 by Disease Severity and Mortality, Southeast United States

\begin{tabular}{|c|c|}
\hline Patient Presentation & No./Total $(\mathrm{N}=502)(\%)$ \\
\hline \multicolumn{2}{|l|}{ Epidemiologic risk factors for COVID-19 } \\
\hline Admitted from congregate setting ${ }^{a}$ & $155 / 502(31)$ \\
\hline Known exposure to COVID-positive individual & $199 / 502(40)$ \\
\hline Healthcare worker & $35 / 502(7)$ \\
\hline Physician & $6 / 35(17)$ \\
\hline Nurse & $11 / 35(31)$ \\
\hline Other & $18 / 35(51)$ \\
\hline \multicolumn{2}{|l|}{ Evaluation location } \\
\hline Patients evaluated prior to admission & $218 / 502(43)$ \\
\hline ED evaluation ${ }^{\mathrm{b}}$ & $133 / 502(61)$ \\
\hline COVID assessment site & $35 / 502(16)$ \\
\hline Other & $50 / 502(23)$ \\
\hline \multicolumn{2}{|l|}{ Testing timing } \\
\hline Patients with positive SARS-CoV-2 assay during admission & $366 / 502(73)$ \\
\hline Mean time to positive test after admission, days & $1.6 \pm 2.9$ \\
\hline Patients with positive SARS-CoV-2 assay prior to admission & $79 / 502(16)$ \\
\hline Mean time to positive test prior to admission, $d$ & $0.7 \pm 2.3$ \\
\hline Not reported & $57 / 502(11)$ \\
\hline \multicolumn{2}{|l|}{ Hospitalized location, state } \\
\hline Arkansas & $56 / 502(11)$ \\
\hline North Carolina & $76 / 502(15)$ \\
\hline Louisiana & $80 / 502(16)$ \\
\hline Mississippi & $127 / 502(25)$ \\
\hline Tennessee & $163 / 502(32)$ \\
\hline \multicolumn{2}{|l|}{ Clinical presentation } \\
\hline Fever (Tmax $>38^{\circ} \mathrm{C}$ in house or prior to admission) & $352 / 502(70)$ \\
\hline Productive cough & $80 / 502(16)$ \\
\hline Dry cough & $277 / 502(55)$ \\
\hline Dyspnea & $317 / 502(63)$ \\
\hline Night sweats & $27 / 502(5)$ \\
\hline Chills & $94 / 502(19)$ \\
\hline Sore throat & $36 / 502(7)$ \\
\hline Fatigue & $187 / 502(37)$ \\
\hline Anorexia & $80 / 502(16)$ \\
\hline Myalgia & $142 / 502(28)$ \\
\hline Nasal congestion & $45 / 502(9)$ \\
\hline Rhinorrhea & $37 / 502(7)$ \\
\hline Nausea & $105 / 502(21)$ \\
\hline Vomiting & $66 / 502(13)$ \\
\hline Diarrhea (>3 episodes of loose stool within previous $24 \mathrm{~h}$ ) & $104 / 502(21)$ \\
\hline Abdominal pain & $49 / 502(10)$ \\
\hline Headache & $70 / 502(14)$ \\
\hline Rash & $2 / 502(0)$ \\
\hline Anosmia (loss of smell) & $25 / 502(5)$ \\
\hline Ageusia (loss of taste) or dysgeusia (altered taste) & $36 / 502(7)$ \\
\hline
\end{tabular}


Table 2. (Continued)

\begin{tabular}{lr}
\hline Patient Presentation & No./Total (N = 502) (\%) \\
\hline Psychataxia (difficulty concentrating) & $29 / 502(6)$ \\
\hline Pleurisy/chest pain & $57 / 502(11)$ \\
\hline No. of patients reporting symptoms prior to admission & $471 / 502(94)$ \\
\hline Mean duration of symptoms prior to admission, $d$ & $5.2 \pm 4.5$ \\
\hline
\end{tabular}

Note. ED, emergency department.

${ }^{a}$ Congregate setting is a school, workplace, prison, etc.

Table 3. Risk of Mortality, Unadjusted and Adjusted Analyses of Hospitalized Patients with COVID-19, Southeast United States

\begin{tabular}{|c|c|c|c|c|}
\hline \multirow[b]{2}{*}{ Covariate } & \multicolumn{2}{|c|}{ Univariate (Unadjusted) } & \multicolumn{2}{|c|}{ Multivariate (Adjusted) } \\
\hline & $\mathrm{OR}^{\mathrm{a}}\left(95 \% \mathrm{Cl}^{\mathrm{b}}\right)$ & $P$ Value & $\mathrm{OR}^{\mathrm{a}}\left(95 \% \mathrm{Cl}^{\mathrm{b}}\right)$ & $P$ Value \\
\hline Age, per decade & $2.13(1.74-2.65)$ & $<.01$ & $2.03(1.56-2.69)$ & $<.01$ \\
\hline Sex, male & $1.81(1.09-3.07)$ & .02 & $2.44(1.34-4.59)$ & $<.01$ \\
\hline \multicolumn{5}{|l|}{ Race/Ethnicity } \\
\hline White & $\operatorname{Ref}^{c}$ & $\ldots$ & $\operatorname{Ref}^{\mathrm{c}}$ & $\ldots$ \\
\hline Black & $0.49(0.30-0.81)$ & $<.01$ & $0.75(0.41-1.36)$ & .34 \\
\hline \multicolumn{5}{|l|}{$\mathrm{BMI}^{\mathrm{d}}$} \\
\hline$<18.5$ & $1.39(0.29-5.22)$ & .65 & $1.34(0.22-6.54)$ & .73 \\
\hline 18.5 to $<25$ & $\operatorname{Ref}^{c}$ & $\ldots$ & $\operatorname{Ref}^{c}$ & $\ldots$ \\
\hline 25 to $<30$ & $0.93(0.46-1.86)$ & .83 & $1.15(0.51-2.64)$ & .73 \\
\hline 30 to $<40$ & $0.55(0.28-1.10)$ & .09 & $1.08(0.48-2.50)$ & .85 \\
\hline$\geq 40$ & $0.85(0.36-1.92)$ & .70 & $1.71(0.58-4.87)$ & .33 \\
\hline \multicolumn{5}{|l|}{ Comorbidities } \\
\hline Cardiovascular disease & $5.01(3.01-8.49)$ & $<.01$ & $2.16(1.15-4.09)$ & .02 \\
\hline Pulmonary disease & $1.52(0.83-2.67)$ & .16 & $1.57(0.78-3.12)$ & .20 \\
\hline Kidney disease & $2.01(1.09-3.57)$ & .02 & $0.89(0.41-1.87)$ & .75 \\
\hline Stroke & $1.81(0.89-3.48)$ & .09 & $1.25(0.55-2.72)$ & .58 \\
\hline Hypertension & $1.83(1.07-3.23)$ & .03 & $0.84(0.42-1.69)$ & .61 \\
\hline Diabetes mellitus & $1.43(0.86-2.37)$ & .16 & $1.43(0.75-2.74)$ & .28 \\
\hline Peripheral vascular & $2.01(0.89-4.22)$ & .07 & $1.25(0.49-3.06)$ & .63 \\
\hline Connective tissue disease & $4.22(1.89-9.21)$ & $<.01$ & $3.13(1.25-7.81)$ & .01 \\
\hline Liver disease & $1.79(0.39-6.15)$ & .39 & $2.10(0.39-8.96)$ & .34 \\
\hline Smoking & $0.87(0.20-2.66)$ & .83 & $1.11(0.22-4.14)$ & .88 \\
\hline Immune compromised & $1.17(0.53-2.34)$ & .68 & $1.58(0.63-3.72)$ & .31 \\
\hline
\end{tabular}

Note. OR, odds ratio; $\mathrm{Cl}$, confidence interval; Ref, reference; BMI, body mass index.

Table 4. Matrix of Mortality Rate by Combined Age/BMI Categories deaths/total (\%) of Hospitalized Patients with COVID-19, Southeast United States

\begin{tabular}{|c|c|c|c|c|c|}
\hline \multirow[b]{2}{*}{ Age Group, y } & \multicolumn{5}{|c|}{ BMI Category, No./Total (\%) } \\
\hline & $<18.5$ & 18.5 to $<25$ & 25 to $<30$ & 30 to $<40$ & $\geq 40$ \\
\hline$<21$ & $0 / 0(0)$ & $0 / 1(0)$ & $0 / 0(0)$ & $0 / 1(0)$ & $0(0)$ \\
\hline $21-40$ & $0 / 0(0)$ & $0 / 10(0)$ & $0 / 12(0)$ & $0 / 31(0)$ & $1 / 13(7.7)$ \\
\hline $41-60$ & $0 / 2(0)$ & $1 / 24(4.2)$ & $2 / 44(4.5)$ & $3 / 72(4.2)$ & $1 / 29(3.4)$ \\
\hline $61-80$ & $0 / 5(0)$ & $9 / 42(21.4)$ & $13 / 60(21.7)$ & $16 / 73(21.9)$ & $9 / 23(39.1)$ \\
\hline$>80$ & $3 / 5(60.0)$ & $8 / 21(38.1)$ & $7 / 15(46.7)$ & $2 / 11(18.2)$ & $0 / 4(0)$ \\
\hline
\end{tabular}

Note. BMI, body mass index. 
Table 5. Risk of Severe Disease, ${ }^{\text {a }}$ Unadjusted and Adjusted Analyses of Hospitalized Patients With COVID-19, Southeast United States

\begin{tabular}{|c|c|c|c|c|}
\hline \multirow[b]{2}{*}{ Covariate } & \multicolumn{2}{|c|}{ Univariate (Unadjusted) } & \multicolumn{2}{|c|}{ Multivariate (Adjusted) } \\
\hline & OR $(95 \% \mathrm{Cl})$ & $P$ Value & OR $(95 \% \mathrm{Cl})$ & $P$ Value \\
\hline Age, per decade & $1.34(1.20-1.52)$ & $<.01$ & $1.17(1.00-1.37)$ & .05 \\
\hline Sex, male & $2.02(1.39-2.94)$ & $<.01$ & $2.34(1.54-3.60)$ & $<.01$ \\
\hline \multicolumn{5}{|l|}{ Race/Ethnicity } \\
\hline White & $\operatorname{Ref}^{c}$ & $\ldots$ & $\operatorname{Ref}^{c}$ & $\ldots$ \\
\hline Black & $0.81(0.56-1.19)$ & .28 & $1.14(0.75-1.76)$ & .54 \\
\hline \multicolumn{5}{|l|}{ BMI } \\
\hline & $0.66(0.14-2.37)$ & .54 & $0.84(0.17-3.31)$ & .81 \\
\hline 18.5 to $<25$ & $\operatorname{Ref}^{\mathrm{C}}$ & $\ldots$ & $\operatorname{Ref}^{\mathrm{C}}$ & $\ldots$ \\
\hline 25 to $<30$ & $1.38(0.80-2.39)$ & .24 & $1.64(0.90-3.04)$ & .11 \\
\hline 30 to $<40$ & $0.97(0.58-1.64)$ & .91 & $1.35(0.75-2.45)$ & .32 \\
\hline$\geq 40$ & $1.19(0.62-2.26)$ & .59 & $1.94(0.93-4.12)$ & .08 \\
\hline \multicolumn{5}{|l|}{ Comorbidities } \\
\hline Cardiovascular disease & $2.52(1.71-3.73)$ & $<.01$ & $1.77(1.09-2.85)$ & .02 \\
\hline Pulmonary disease & $0.90(0.56-1.44)$ & .67 & $0.87(0.51-1.48)$ & .62 \\
\hline Kidney disease & $1.40(0.86-2.26)$ & .17 & $0.86(0.48-1.51)$ & .60 \\
\hline Stroke & $1.37(0.78-2.38)$ & .27 & $0.97(0.51-1.81)$ & .93 \\
\hline Hypertension & $1.43(0.98-2.09)$ & .06 & $1.08(0.67-1.74)$ & .75 \\
\hline Diabetes mellitus & $1.35(0.92-1.98)$ & .12 & $1.19(0.75-1.87)$ & .46 \\
\hline Peripheral vascular & $1.46(0.74-2.83)$ & .27 & $1.07(0.50-2.25)$ & .87 \\
\hline Connective tissue disease & $2.12(1.03-4.44)$ & .04 & $1.68(0.76-3.71)$ & .20 \\
\hline Liver disease & $2.58(0.85-8.64)$ & .10 & $2.54(0.79-8.93)$ & .12 \\
\hline Smoking & $1.33(0.55-3.13)$ & .52 & $1.53(0.58-3.92)$ & .38 \\
\hline Immune compromised & $1.58(0.92-2.69)$ & .10 & $1.76(0.97-3.18)$ & .06 \\
\hline
\end{tabular}

Note. OR, odds ratio; $\mathrm{Cl}$, confidence interval; Ref, reference; $\mathrm{BMI}$, body mass index.

a Severe disease, defined as a composite of ICU admission or requirement of mechanical ventilation.

Most inpatients with COVID-19 in our study were obese, with a median BMI of 30.5, consistent with previous studies demonstrating increased rates of obesity among those hospitalized with COVID-19. ${ }^{7,21,22}$ In our study, obesity was not an independent predictor of severe disease or mortality in a multivariate analysis after controlling for other comorbidities such as diabetes and cardiovascular disease. In contrast, previous studies found morbid obesity (BMI > 40) to be associated with increased risk of COVID-19 critical illness. $^{23}$

Racial disparities among patients diagnosed with COVID-19 in the United States have been widely publicized. One study found a positive correlation between the prevalence of racial minorities and COVID-19 case and mortality rates; however, these results were not adjusted for potential confounders. ${ }^{24}$ Multiple case series have shown that self-identified African American, black, or black non-Hispanic race is an independent risk factor for hospital admission. ${ }^{13,14,25}$ A large retrospective cohort in Louisiana found that black, non-Hispanic patients accounted for $70.4 \%$ of COVID-19 cases, $76.9 \%$ of hospital admissions, and $70.6 \%$ of deaths despite constituting only $31 \%$ of the total health system population. ${ }^{14}$ Consistent with our findings, those investigators found that race was not independently associated with mortality after adjusted analysis.

Most of our patients self-identified as either Caucasian (white) or African American (black). Only 5\% identified as Asian or "other," and Hispanic ethnicity was infrequently reported limiting robust analysis for these populations. Data were manually abstracted using chart review and did not rely on automatic abstraction, which provides confidence in these results. Our analysis included patients from the initial phase of the pandemic and does not account for the changing demographics of those hospitalized with COVID-19 in the Southeast over time. Personal communications at each of the sites note a larger proportion of Hispanic patients now hospitalized, with increased community spread and localized outbreaks identified in certain industries (eg, food and meat processing). Our study was restricted to the first 10 weeks of the pandemic in the Southeast, before widespread community prevalence. This factor may account for the low Hispanic population and older age of our study cohort, despite the multicenter design.

We found that blacks represented a large portion of COVID-19 hospital admissions (57.2\%) despite comprising only $16.1 \%-37.6 \%$ of the population in the states represented in this study. ${ }^{26}$ Univariate analysis suggested increased in-hospital mortality among white patients despite no difference in rates of severe disease. However, this finding lost statistical significance after controlling for age and comorbid conditions. The CDC has suggested that the increased risk of hospitalization among racial minorities may be due to living, work, and health circumstances including a greater burden of concomitant comorbidities such as diabetes, 


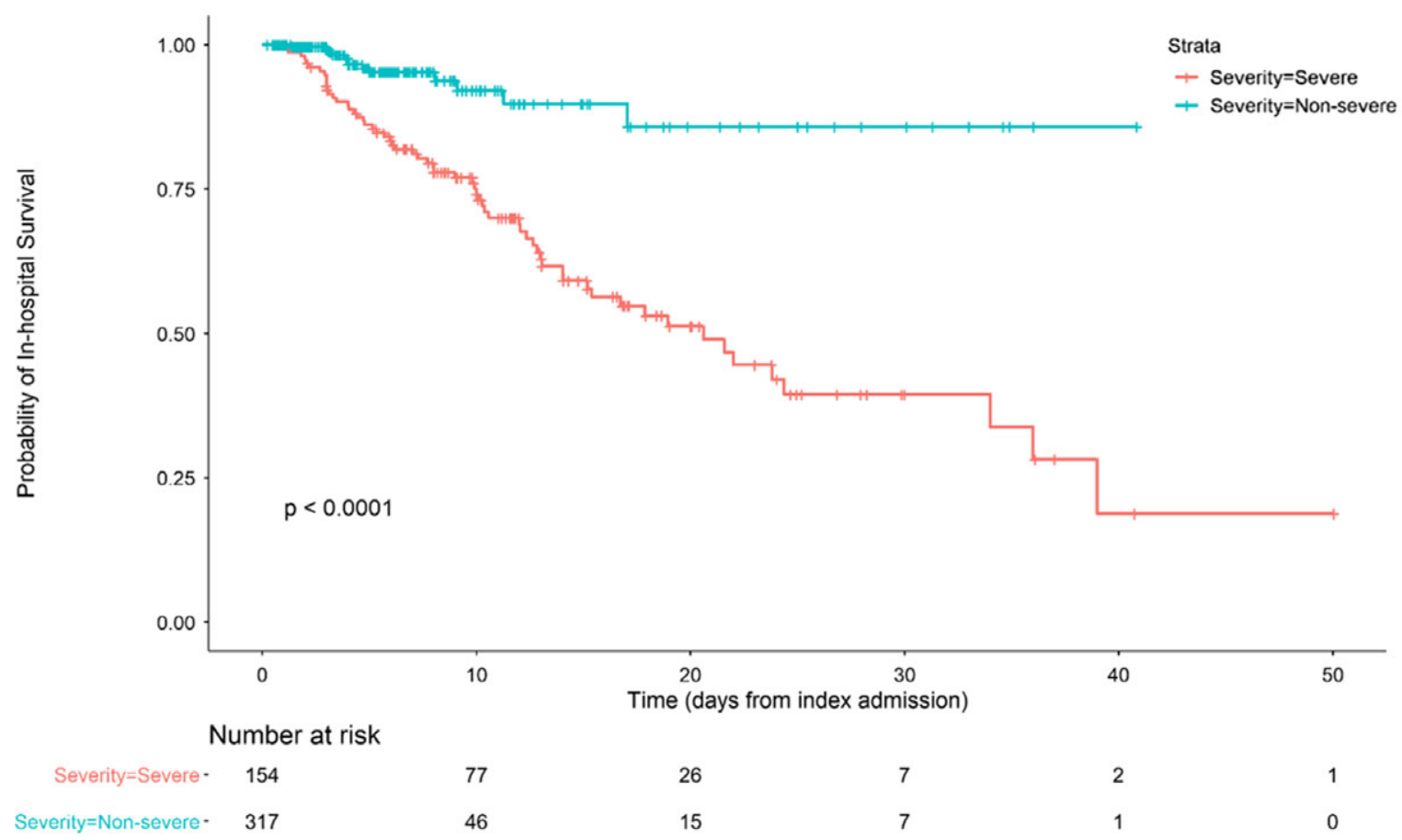

Fig. 1. Kaplan-Meier curve stratified by disease severity. Severe disease is defined as a composite of ICU admission or requirement of mechanical ventilation.

hypertension, and obesity. ${ }^{27}$ Other potential hypotheses include differential employment in essential jobs, reliance on public transportation, and residence in Southern states where "shelter at home" orders were delayed or prematurely lifted. ${ }^{28}$

Our results do not demonstrate any significant correlation between smoking, asthma, or COPD and increased mortality or disease severity from COVID-19, though we recognize the small overall sample sizes for each being $4 \%, 10 \%$, and $7 \%$, respectively (Supplementary Tables S1 and S2 online). Previous studies have yielded conflicting results regarding the association between smoking and COVID-19 disease severity or death. ${ }^{29,30}$ Interestingly, some have hypothesized various explanations for these observations ranging from modulation of pathogenesis to differential practice of preventive measures. ${ }^{31-33}$ These explanations should be interpreted with caution given the inability to establish causality.

History of cardiovascular disease was a significant risk factor in COVID-19 similar to prior studies, more than doubling the risk for mortality and nearly doubling the risk for severe disease. ${ }^{3,34}$ Pre-existing cardiovascular disease has been associated with worse clinical outcomes in patients who develop COVID-19. ${ }^{35}$ Interestingly, although hypertension was the most common comorbidity reported and appeared to increase risk of mortality in univariate analysis, this finding did not persist in the multivariate analysis. Contradictory to some previous studies, neither our univariate nor our multivariate analysis showed that hypertension increased risk of severe COVID-19.,29

This study has several limitations, mainly due to the retrospective design. They include the evolving nature of the characteristics of the SARS-CoV-2 outbreak during the period of consecutive patient data collection, limited availability of testing, and variability of treatment algorithms across sites. The timing of the outbreak was delayed in some of the Southeast states compared to other states, and each hospital population represents only the initial population of hospitalized COVID-19 patients likely before extensive community spread. This phenomenon may have skewed the study toward more vulnerable populations including those in long-term care facilities and other congregate settings where early spread was recognized. However, the multistate design including communitybased and tertiary-care hospital representation provided broad representation across the Southeast region of the United States and limited bias toward a single group. We did not collect data on initial oxygen requirement that, in retrospect, could help identify patients who might require mechanical ventilation. Additionally, the reduced availability of COVID-19 testing at the beginning of the pandemic, and potential underreporting and misclassification of COVID-19 cases attributed to other circulating seasonal respiratory viruses, may have influenced complete case ascertainment. All sites used FDA-approved molecular diagnostic tests to perform RT-PCR; however, the different RNA gene targets used to identify SARS-CoV-2 can vary in their sensitivity ${ }^{36,37}$ Furthermore, small sample size in some of the stratified analysis may have limited our ability to detect a difference between groups, such as individual underlying conditions (eg, COPD). The loss of effect upon multivariate adjustment likely suggests the results being underpowered and the presence of some confounding between comorbidities. The absence of a clear link between obesity and mortality in our study does not rule out such a possibility. Rather, because of the high prevalence of obesity overall and the low number of subjects and events occurring at more extreme BMIs, our study may simply have been underpowered to detect such an effect. There is also likely high variability in treatments administered in the beginning of this pandemic due to the lack of approved treatments available. However, this limitation is likely minimal because few treatment options with proven clinical benefit, such as remdesivir, were widely available at the time of data collection for this study.

This multicenter cohort provides a robust analysis adjusting for potential confounders to describe risk factors associated with hospital mortality and severe disease from COVID-19 in the Southeast region of the United States. Increasing and advanced age, male sex, and cardiovascular disease are all independent risk factors for mortality and progression to severe disease. Furthermore, mortality 
risk doubles with each successive decade of life. Notably, neither obesity nor race were significantly associated with either outcome after adjusted analyses. Understanding patients at risk offers vital information as the region braces for increased healthcare utilization, informs critical resource needs, and emphasizes awareness of patients at risk for severe clinical outcomes.

Acknowledgments. We thank the administrations of each health-system and institution for administrative and information technology support.

Financial support. No financial support was provided relevant to this article.

Conflicts of interest. All authors report no conflicts of interest relevant to this article.

Supplementary material. To view supplementary material for this article, please visit https://doi.org/10.1017/ice.2020.1435

\section{References}

1. WHO Director-General's opening remarks at the media briefing on COVID-19. World Health Organization website. https://www.who.int/dg/ speeches/detail/who-director-general-s-opening-remarks-at-the-mediabriefing-on-covid-19-11-march-2020. Accessed April 1.

2. Huang C, Wang Y, Li X, et al. Clinical features of patients infected with 2019 novel coronavirus in Wuhan, China. Lancet 2020;395:497-506.

3. Yang J, Zheng Y, Gou X, et al. Prevalence of comorbidities and its effects in patients infected with SARS-CoV-2: a systematic review and meta-analysis. Int J Infect Dis 2020;94:91-95.

4. Guan W-J, Liang W-H, Zhao Y, et al. Comorbidity and its impact on 1590 patients with COVID-19 in China: a nationwide analysis. Eur Respir J 2020;55:2000547.

5. Guo T, Fan Y, Chen M, et al. Cardiovascular implications of fatal outcomes of patients with coronavirus disease 2019 (COVID-19). JAMA Cardiol 2020;5(7):1-8.

6. Zhou F, Yu T, Du R, et al. Clinical course and risk factors for mortality of adult inpatients with COVID-19 in Wuhan, China: a retrospective cohort study. Lancet 2020;395:1054-1062.

7. Richardson S, Hirsch JS, Narasimhan M, et al. Presenting characteristics, comorbidities, and outcomes among 5,700 patients hospitalized with COVID-19 in the New York City area. JAMA 2020;323:2052-2059.

8. Myers LC, Parodi SM, Escobar GJ, et al. Characteristics of hospitalized adults with COVID-19 in an integrated health care system in California. JAMA 2020;323:2195-2198.

9. Current cigarette smoking among adults in the United States. Centers for Disease Control and Prevention website. https://www.cdc.gov/tobacco/data statistics/fact_sheets/adult_data/cig_smoking/index.htm. Updated December 21, 2020. Accessed January 6, 2020.

10. Diabetes and obesity maps. Centers for Disease Control and Prevention website. https://www.cdc.gov/diabetes/data/center/slides.html. Accessed January 6, 2021.

11. Hypertension maps and data sources. Centers for Disease Control and Prevention website. https://www.cdc.gov/bloodpressure/maps_data.htm. Accessed January 6, 2021.

12. Adult obesity prevalence maps. Centers for Disease Control and Prevention website. https://www.cdc.gov/obesity/data/prevalence-maps.html. Accessed April 1, 2020.

13. Killerby ME, Link-Gelles R, Haight SC, et al. Characteristics associated with hospitalization among patients with COVID-19-Metropolitan Atlanta, Georgia, March-April 2020. Morb Mortal Wkly Rep 2020;69:790-794.

14. Price-Haywood EG, Burton J, Fort D, et al. Hospitalization and mortality among black patients and white patients with COVID-19. N Engl J Med 2020;382:2534-2543.

15. Symptoms of coronavirus. Centers for Disease Control and Prevention website. https://www.cdc.gov/coronavirus/2019-ncov/symptoms-testing/symptoms. html?CDC_AA_refVal=https\%3A\%2F\%2Fwww.cdc.gov\%2Fcoronavirus\% 2F2019-ncov\%2Fabout\%2Fsymptoms.html. Accessed July 8, 2020.
16. Harris PA, Taylor R, Thielke R, et al. Research electronic data capture (REDCap) - a metadata-driven methodology and workflow process for providing translational research informatics support. J Biomed Inform 2009;42:377-381.

17. Information for laboratories about coronavirus (COVID-19). Centers for Disease Control and Prevention website. https://www.cdc.gov/coronavirus/ 2019-nCoV/lab/guidelines-clinical-specimens.html\#specimen. Accessed June 21, 2020.

18. Iaccarino G, Grassi G, Borghi C, et al. Age and multimorbidity predict death among COVID-19 patients. Hypertension 2020;76:366-372.

19. Imam Z, Odish F, Gill I, et al. Older age and comorbidity are independent mortality predictors in a large cohort of 1,305 COVID-19 patients in Michigan, United States. J Intern Med 2020. doi: 10.1111/joim.13119.

20. Zhao M, Wang M, Zhang J, et al. Comparison of clinical characteristics and outcomes of patients with coronavirus disease 2019 at different ages. Aging 2020;12:10070-10086.

21. Lighter J, Phillips M, Hochman S, et al. Obesity in patients younger than 60 years is a risk factor for COVID-19 hospital admission. Clin Infect Dis 2020: ciaa415.

22. Simonnet A, Chetboun M, Poissy J, et al. High prevalence of obesity in severe acute respiratory syndrome coronavirus-2 (SARS-CoV-2) requiring invasive mechanical ventilation. Obesity 2020;28:1195-1199.

23. Petrilli CM, Jones SA, Yang J, et al. Factors associated with hospitalization and critical illness among 5279 patients with COVID-19 disease in New York City: a prospective cohort study. BMJ 2020 May 22;369: m1966.

24. Mahajan UV, Larkins-Pettigrew M. Racial demographics and COVID-19 confirmed cases and deaths: a correlational analysis of 2,886 US counties. J Public Health 2020: fdaa070.

25. Suleyman G, Fadel RA, Malette KM, et al. Clinical characteristics and morbidity associated with coronavirus disease 2019 in a series of patients in metropolitan Detroit. JAMA Netw Open 2020;3:e2012270-e.

26. Rastogi SJT, Hoeffel EM, Drewery MP. The black population: 2010 census briefs. https://www.census.gov/prod/cen2010/briefs/c2010br-06.pdf. Accessed May 8, 2020.

27. COVID-19 in racial and ethnic minority groups. Centers for Disease Control and Prevention website. https://www.cdc.gov/coronavirus/2019ncov/need-extra-precautions/racial-ethnic-minorities.html. Accessed June 29, 2020.

28. Dyer O. COVID-19: black people and other minorities are hardest hit in the US. BMJ 2020: $\mathrm{m} 1483$.

29. Guan WJ, Ni ZY, Hu Y, et al. Clinical characteristics of coronavirus disease 2019 in China. N Engl J Med 2020;382:1708-1720.

30. Lippi G, Henry BM. Active smoking is not associated with severity of coronavirus disease 2019 (COVID-19). Eur J Intern Med 2020;75: 107-108.

31. Bruine de Bruin W, Bennett D. Relationships between initial COVID-19 risk perceptions and protective health behaviors: a national survey. Am J Prev Med 2020;59:157-167.

32. Miyara M, Tubach F, Pourcher V, et al. Low incidence of daily active tobacco smoking in patients with symptomatic COVID-19. Qeios 2020. doi: 10.32388/WPP19W.3.

33. Oakes JM, Fuchs RM, Gardner JD, et al. Nicotine and the reninangiotensin system. Am J Physiol Regul Integr Comp Physiol 2018;315: R895-R906.

34. Madjid M, Safavi-Naeini P, Solomon SD, et al. Potential effects of coronaviruses on the cardiovascular system: a review. JAMA Cardiol 2020;5: 831-840.

35. Bansal M. Cardiovascular disease and COVID-19. Diabetes Metab Syndr 2020;14:247-250.

36. In vitro diagnostics EUAs. Food and Drug Administration website. https:// www.fda.gov/medical-devices/coronavirus-disease-2019-covid-19-emergencyuse-authorizations-medical-devices/vitro-diagnostics-euas. Accessed July $17,2020$.

37. Nalla AK, Casto AM, Huang M-LW, et al. Comparative performance of SARS-CoV-2 detection assays using seven different primer-probe sets and one assay kit. J Clin Microbiol 2020;58:e0557-20. 